in vivo $34: 1685-1694(2020)$

doi:10.21873/invivo.11962

\title{
The Effect of Coronavirus (COVID-19) on Breast Cancer Teamwork: A Multicentric Survey
}

\author{
GIANLUCA VANNI $^{1}$, MARCO MATERAZZO ${ }^{1}$, FRANCESCA SANTORI $^{1}$, MARCO PELLICCIARO $^{1}$, \\ MARIA COSTESTA $^{1}$, PAOLO ORSARIA ${ }^{2}$, FRANCESCA CATTADORI $^{3}$, CHIARA ADRIANA PISTOLESE ${ }^{4}$, \\ TOMMASO PERRETTA ${ }^{4}$, MARCELLO CHIOCCHI ${ }^{4}$, ROSARIA MEUCCI ${ }^{1,4}$, FELICIANA LAMACCHIA ${ }^{4}$, \\ MASSIMO ASSOGNA ${ }^{1}$, , JONATHAN CASPI ${ }^{1}$, ALESSANDRA VITTORIA GRANAI ${ }^{1}$, ADRIANO DE MAJO ${ }^{1}$, \\ AGOSTINO CHIARAVALLOTI ${ }^{5,6}$, MARIA ROLANDO D'ANGELILLO ${ }^{7}$, ROSARIA BARBARINO $^{7}$, \\ SARA INGALLINELLA ${ }^{1}$, LJUBA MORANDO ${ }^{1}$, STEFANIA DALLI ${ }^{1}$, ILARIA PORTARENA ${ }^{8}$, \\ VITTORIO ALTOMARE ${ }^{2}$, GIOVANNI TAZZIOLI ${ }^{9}$ and ORESTE CLAUDIO BUONOMO ${ }^{1}$ \\ ${ }^{1}$ Breast Unit, Department of Surgical Science, Policlinico Tor Vergata University, Rome, Italy; \\ ${ }^{2}$ Department of Breast Surgery, University Campus Bio-Medico, Rome, Italy; \\ ${ }^{3}$ UO Breast Surgery, Breast Unit, "Guglielmo da Saliceto" Hospital, Piacenza, Italy; \\ ${ }^{4}$ Department of Diagnostic Imaging and Interventional Radiology, Molecular \\ Imaging and Radiotherapy, Policlinico Tor Vergata University, Rome, Italy; \\ ${ }^{5}$ Department of Biomedicine and Prevention, Policlinico Tor Vergata University, Rome, Italy; \\ ${ }^{6}$ IRCCS Neuromed, UOC Medicina Nucleare, Pozzilli, Italy; \\ ${ }^{7}$ Radiotherapy Unit, Department of Oncology and Hematology, Policlinico Tor Vergata University, Rome, Italy; \\ ${ }^{8}$ Department of Oncology, Policlinico Tor Vergata University, Rome, Italy; \\ ${ }^{9}$ Oncologic Breast Surgery Unit, Azienda Ospedaliero-Universitaria Policlinico Hospital of Modena, Modena, Italy
}

\begin{abstract}
Background/Aim: Despite the large amount of clinical data available of Coronavirus-19 (COVID-19), not many studies have been conducted about the psychological toll on Health Care Workers (HCWs). Patients and Methods: In this multicentric descriptive study, surveys were distributed among 4 different Breast Cancer Centers (BCC). BCCs were distinguished according to COVID-19 tertiary care hospital (COVID/No-COVID) and district prevalence (DP) (High vs. Low). DASS-21 score, PSS score and demographic data (age, sex, work) were evaluated. Results: A total of $51 \mathrm{HCWs}$ were analyzed in the study. Age, work and sex did not demonstrate statistically significant values. Statistically significant distribution was found between DASS-21-stress score and COVID/No-COVID $(p=0.043)$. No
\end{abstract}

This article is freely accessible online.

Correspondence to: Materazzo Marco, Breast Unit, Department of Surgical Science, PTV: Policlinico Tor Vergata University, Viale Oxford 81, 00133 Rome, Italy. Tel: +39 3395685883, e-mail: mrcmaterazzo@gmail.com

Key Words: COVID-19, breast cancer, SARS-SOV-2, DASS-21, PSS, score, anxiety, heath care workers, psychological well- being, multicentric study. difference was found in the remaining DASS-21 and PSS scores, dividing the HCWs according to COVID-19-hospital and DP. Conclusion: Working in a COVID-19-hospital represents a factor that negatively affects psychosocial wellbeing. However, DP seems not to affect the psychosocial well-being of BCC HCWs. During the outbreak, psychological support for low risk $\mathrm{HCW}$ should be provided regardless $D P$.

The first case of atypical pneumonia previously named as Severe Acute Respiratory Syndrome Corona Virus 2 (COVID-19) was recorded in December 2019 in China. Italy has been the first European country to be involved in this outbreak. The national health care system (NHS) is currently reallocating resources from elective and semi-elective patients toward severe COVID-19 patients $(1,2)$. Italy has exceeded the cases recorded in China and unfortunately, up to now (May, 17th), 225,435 cases and 31908 deaths have been registered with still over 700 admitted to intensive care units (3). The Italian outbreak is mainly located in clusters along the north of Italy (Lombardia, Veneto and Emilia Romagna) and a low incidence in central and southern regions $(1,3,4)$.

In order to control COVID-19, resource reallocation eventually led to the creation of hospitals partially or totally dedicated to COVID-19 patients (COVID-hospital), therefore 
Table I. Distribution of Breast Centre according to COVID-19 prevalence and COVID-hospital designation.

\begin{tabular}{lcc}
\hline & Low prevalence & High prevalence \\
\hline COVID Hospital & Policlinico Tor Vergata University Hospital & "Guglielmo da Saliceto" Hospital Piacenza \\
No-COVID Hospital & Campus Bio-Medico University Hospital of Rome & Azienda Ospedaliero-Universitaria Policlinico Hospital of Modena \\
\hline
\end{tabular}

increasing numbers of health care workers (HCWs) have been facing a new highly contagious infectious disease (5). In spite of having a separate route and applying infection control (IC) measures during this period, patients could refuse surgery or diagnostic procedures due to COVID-19 infection risk, reducing the number of admitted patients in the hospital (2, 6-8). Moreover, IC measures include instructions to $\mathrm{HCW}$ s to maintain a physical distance from other colleagues, patients and families which could eventually lead to psychological distress (9-11). Previous studies that took place during the Toronto SARS outbreak have shown that many HCWs presented high levels of psychological distress who were mostly concerned about infecting family and friends as well as about the disease consequences on their own health. Worries about their functional ability and fear of stigmatization along with social isolation and higher job stress have been described (9-11). Many studies showed HCWs who are in the front line of the diagnosis, treatment and care of patients with an emerging disease like COVID-19, are more frequently at risk of developing psychological distress and mental health disorders (12-15). Other causes of HCWs' psychological distress were strenuous workload, depletion of personal protective equipment, lack of specific drugs and the uncertainty of dealing with an illness whose transmissibility is partially understood (15).

Despite the great deal of existing literature regarding psychological distress in the high-risk area, little is known about the psychological distress among HCWs at low risk and the impact of the outbreak on HCWs who aren't directly involved in COVID-19 treatment. The present study aimed to assess differences in the degree of depression, anxiety and distress among different Italian Breast Cancer Centers (BCCs) HCWs according to COVID hospital/No-COVID hospital and prevalence of the disease in the hospital district (high prevalence $v s$. low prevalence).

\section{Patients and Methods}

Study design. The study was designed as multicentric and descriptive. The institutional review board of Policlinico Tor Vergata waived the need for a formal approval. Due to the difference in COVID-19 prevalence (4) and national breast screening programs across Italy $(13,14)$, we included 4 different BCCs across Italy: Azienda Ospedaliero-Universitaria Policlinico Hospital of Modena,
Campus Bio-Medico University Hospital of Rome, "Guglielmo da Saliceto" Hospital Piacenza and Policlinico Tor Vergata University Hospital. BCCs and Hospital were divided as shown in Table I. COVID-19 hospital was defined as the hospital that was totally or partially designated by the NHS as tertiary care institutions for COVID-19 disease.

Population. All HCWs from 4 Italian BCCs were invited to complete written questionnaires between April $1^{\text {st }}$ and April 30 $3{ }^{\text {th }} ; 51$ forms were collected. The main inclusion criteria were work in tertiary BCCs and no involvement with COVID-19 patients. All participants were allowed to terminate the survey at any time they wished. The population was subdivided for analysis according to demographic data, prevalence (high prevalence vs. low prevalence) and COVID hospital (COVID vs. No-COVID hospital). HCWs' roles were recorded and divided between physician, surgeon, nurse.

Questionnaires and score. An e-mail was sent to each breast cancer unit manager, informing about the study and asking for their permission to distribute the questionnaires among their employees. Blank anonymous questionnaires were placed at various designated work areas in each participating unit during the study period. All questionnaires were collected by personnel not involved in the study analysis from each BCC and were sent to Tor Vergata for analysis. To assess HCW's worries and concerns over the pandemic, their behavior during the outbreak and whether these factors were related to psychological distress and other mental health symptoms like depression, anxiety and stress, the following two self-reported questionnaires were administered: the depression, anxiety and stress scale (DASS-21) and the perceived stress scale (PSS) by Sheldon Cohen. The first part of both questionnaires' inquiries about basic demographic data (age, sex, occupation and workplace).

The depression, anxiety and stress scale -21 items (DASS-21) is a set of three self-report scales designated to measure the emotional states of depression, anxiety and stress. Each of the three DASS-21 scales contains 7 items, divided into subscales with similar content. Participants were asked to respond according to how closely the item applied to them in the period of the outbreak. The scale uses the Likert four-level scoring system, with 0 to 3 points representing nonconformity (0) to very consistent (3). Higher scores are linked with higher level of negative emotions (14).

The total scores of these measurement tools were interpreted as follows: Depression, normal [0-9], mild [10-13], moderate [14-20], severe [21-27], extremely severe (28+); Anxiety, normal [0-7], mild [8-9], moderate [10-14], severe [15-19], extremely severe [20+]; Stress, normal [0-14], mild [15-18], moderate [19-25], severe [2633], extremely severe [34+].

The 10-item Perceived Stress Scale (PSS) by Sheldon Cohen (16) is the most widely used psychological instrument for measuring global perceived stress and is a robust predictor of health and disease $(13,17)$. The scale measures global perceived stress 
Table II. Demographic characteristics, Mean DASS-21 and PSS score, Subdivision according DASS-21 and PSS score subdivision. Categorical variables are expressed as numbers and percentages, continuous variables are expressed as a median and an IQR (interquartile range).

\begin{tabular}{lc}
\hline Demographics & \\
\hline Age [median (IQR)] & $47(40-54) \mathrm{yr}$ \\
Female gender, N (\%) & $30 / 46(65.22 \%)$ \\
Prevalence distribution & \\
$\quad$ Low prevalence N (\%) & $27 / 46(58.70 \%)$ \\
High prevalence N (\%) & $19 / 46(41.30 \%)$ \\
COVID Hospital & \\
NO COVID Hospital N (\%) & $17 / 46(37.00 \%)$ \\
COVID Hospital N (\%) & $29 / 46(63.00 \%)$ \\
Profession, N (\%) & \\
Nurse & $10 / 46(21.73 \%)$ \\
Physician & $18 / 46(39.13 \%)$ \\
Surgeon & $18 / 46(39.13 \%)$ \\
DASS-21 Score & \\
Stress [median (IQR)] & $16(10-21.5)$ \\
Anxiety [median (IQR)] & $5(2.5-11.5)$ \\
Depression [median (IQR)] & $6(4-13.5)$ \\
Stress N (\%) & \\
Normal & $13 / 46(28.26 \%)$ \\
Mild to moderate & $27 / 46(58.70 \%)$ \\
Severe to extremely severe & $6 / 46(13.04 \%)$ \\
Anxiety N (\%) & \\
Normal & $28 / 46(60.87 \%)$ \\
Mild to moderate & $12 / 46(19.56 \%)$ \\
Severe to extremely severe & $6 / 46(13.04 \%)$ \\
Depression N (\%) & \\
Normal & $29 / 46(63.04 \%)$ \\
Mild to moderate & $9 / 46(19.56 \%)$ \\
Severe to extremely severe & $8 / 46(17.39 \%)$ \\
PSS Score & \\
PSS score [median (IQR)] & $16.5(11.25-21)$ \\
PSS Score & \\
Low perceived stress N (\%) & $15 / 46(32.61 \%)$ \\
Medium perceived stress N (\%) & $26 / 46(56.52 \%)$ \\
High perceived stress N (\%) & $5 / 46(10.87 \%)$ \\
\hline & \\
& \\
\hline &
\end{tabular}

experienced across the past 30 days on a 5-point scale: never [0], almost never [1], once in a while [2], often [3], very often [4]. Total scores range from 0 to 40 . Total PSS score is classified as: 0-13 low perceived stress, 14-26 medium perceived stress, 27-40 high perceived stress.

Statistical analysis. All data were submitted into the EXCEL datasheet (Microsoft, Washington, DC, USA). DASS-21 and PSS10 scores were analyzed as continuous variables and ordinal variables according to the respective classification. Due to the small sample size, all continues variables are expressed as a median and an interquartile range (IQR). $t$-test and ANOVA were used to determine the $p$-value for continuous variables. When DASS-21 score was treated as dummy variable, independent-samples KruskalWallis test or Independent-Samples Mann-Whitney $U$-test were used to determine the $p$-value for nominal data or dichotomous variables, respectively. Pearson's coefficient was used to assess any correlation
Table III. Linear correlation and DASS-21 and PSS scores. All Values are reported as a median and an IQR in the brackets. IQR: Interquartile range; R: Pearson coefficient.

\begin{tabular}{lcccc}
\hline & $\begin{array}{c}\text { DASS-21 } \\
\text { stress }\end{array}$ & $\begin{array}{c}\text { DASS-21 } \\
\text { anxiety }\end{array}$ & $\begin{array}{c}\text { DASS-21 } \\
\text { depression }\end{array}$ & PSS-10 \\
\hline Median (IQR) & $\begin{array}{c}16(10-21.5) \\
\text { R }\end{array}$ & $\begin{array}{c}5(2.5-11.5) \\
6(4-13.5)\end{array}$ & $\begin{array}{c}16.5(11.25-21) \\
0.1853\end{array}$ \\
\hline
\end{tabular}

between continuous variables and PSS-10 and DASS-21 scores. Categorical data were recoded as numbers and percentages. Analysis was performed using the Fisher's exact test for dichotomous variable. All the statistical analyses were performed in SPSS statistical package version 23.0 (SPSS Inc., Chicago, IL, USA).

\section{Results}

Demographic data. A total of $51 \mathrm{HCW}$ s answered to the survey, 5 HCWs were excluded from the study as they didn't complete the demographic data or both tests (PSS-10 or DASS-21). Thus, the population consisted of $46 \mathrm{HCWs}$ from the 4 different hospitals. $65.22 \%$ of the HCWs who answered the survey were females. $78.26 \%$ were medical doctors and the remaining nurses $(21.74 \%)$. HCWs' population was subsequently divided according to COVID/NO-COVD and prevalence. Twenty-nine $(63 \%)$ of the surveyed population worked in COVID hospital and 17 (37\%) in No-COVID hospital. Grouping by districts, $27 \mathrm{HCWs}$ (58.70\%) worked in low prevalence areas and $19(41.30 \%)$ in high prevalence districts (Table II).

In the general population, DASS-21 score revealed a median value of 16,5 , and 6 regarding stress, anxiety and depression, respectively. $71.74 \%$ of HCWs experienced mild to extremely severe stress level according to DASS-21 score. $32.6 \%$ HCWs experienced anxiousness and $36.95 \%$ depression symptoms. Median PSS score was 16.5. Table III summarizes calculations of linear regression between DASS21 and PSS-10 scores with age, showing no correlations (R: $0.0217,-0.033,0.2424,0.1853$ for DASS-21 Stress, DASS21 Anxiety, DASS-21 depression and PSS-10, respectively).

Subdivision of the population according to sex exhibited no difference in terms of median DASS-21 stress, anxiety and depression values. Moreover, PSS scores demonstrated casual distribution between ages (Table IV).

Analyzing data regarding Profession and DASS-21 or PSS score value showed no statistical significance. Population grouping according to the level of symptoms (normal, mild, moderate, severe, extremely severe for DAS-21 score and low, medium and high perceived stress) didn't show any statistical difference in stress, anxiety and depression or perceived stress level among nurses, physicians and surgeons (Table V). 
Table IV. DAS-21 score and PSS score according to Sex. All values are express as medians and IQRs (interquartile ranges). All categorical data are expressed as numbers and percentages. $p$-Values are obtained with T-test for continuous variable and with Independent-Samples Mann-Whitney U-Test for ordinal data.

\begin{tabular}{|c|c|c|c|}
\hline & Male $(n=16)$ & Female $(n=30)$ & $p$-Value \\
\hline DASS-21 stress & $16[10.5 ; 20]$ & $14[10 ; 22]$ & 0.773 \\
\hline DASS-21 anxiety & $8[2 ; 12.5]$ & $4[4 ; 8]$ & 0.370 \\
\hline DASS-21 depression & $7[2 ; 13]$ & $6[4.5 ; 13]$ & 0.999 \\
\hline PSS-10 & $15[10.5 ; 21.5]$ & $17[12.25 ; 20.75]$ & 0.873 \\
\hline \multicolumn{4}{|l|}{ DASS-21 Stress } \\
\hline Normal & $4(25 \%)$ & $9(30 \%)$ & 0.808 \\
\hline Mild & $7(43.75 \%)$ & $11(36.77 \%)$ & \\
\hline Moderate & $2(12.5 \%)$ & $7(23.33 \%)$ & \\
\hline Severe & $1(6.25 \%)$ & $1(3.33 \%)$ & \\
\hline Extremely severe & $2(12.5 \%)$ & $2(6.67 \%)$ & \\
\hline \multicolumn{4}{|l|}{ DASS-21 anxiety } \\
\hline Normal & $8(50 \%)$ & $20(66.67 \%)$ & 0.700 \\
\hline Mild & $0(0 \%)$ & $4(13.33 \%)$ & \\
\hline Moderate & $5(31.25 \%)$ & $2(6.67 \%)$ & \\
\hline Severe & $1(6.25 \%)$ & $0(0 \%)$ & \\
\hline Extremely severe & $2(12.5 \%)$ & $3(10 \%)$ & \\
\hline \multicolumn{4}{|l|}{ DASS-21 depression } \\
\hline Normal & $11(68.75 \%)$ & $18(60 \%)$ & 0.576 \\
\hline Mild & $3(18.75 \%)$ & $2(6.67 \%)$ & \\
\hline Moderate & $0(0 \%)$ & $4(13.33 \%)$ & \\
\hline Severe & $0(0 \%)$ & $4(13.33 \%)$ & \\
\hline Extremely severe & $2(12.5 \%)$ & $2(6.67 \%)$ & \\
\hline \multicolumn{4}{|l|}{ PSS } \\
\hline Low perceived stress & $6(37.5 \%)$ & $9(30 \%)$ & 0.927 \\
\hline Medium perceived stress & $7(43.75 \%)$ & $19(63.33 \%)$ & \\
\hline High perceived stress & $3(18.75 \%)$ & $2(6.67 \%)$ & \\
\hline
\end{tabular}

COVID No-COVID analysis. HCWs population was subsequently divided according to COVID/No-COVID hospital. As mentioned before in Table I, Piacenza-Parma Hospital and Roma Tor Vergata Breast Cancer Center were designated as COVID hospitals by the National Health system in low prevalence and high prevalence areas, respectively. Campus Bio-Medico University Hospital and Modena University Hospital were used as control for this analysis in low and high prevalence areas, respectively.

Consequently, 29 (63\%) questionnaires were collected from COVID hospital and $17(23 \%)$ were submitted from NoCOVID hospital. As displayed in Table VI, the analysis did not identify any significant differences in the distribution of DASS-21 subset of anxiety and depression and PSS score in the COVID hospital compared to No-COVID hospital $(p>0.05)$. Conversely, statistically significant distribution was found in the median DASS-21 score $(p=0.043)$. However, independent-samples Mann-Whitney $U$-test failed to find statistically significant distribution between the two cohorts $(p>0.05)$ in regard to the DASS-21 stress score according to groups (normal, mild, moderate, severe, extremely severe).
COVID Hospital HCWs exhibited lower mean value of reported stress when compared with No-COVID hospital HCWs (22.03 vs. 26.00). This result was confirmed when the PSS scores were classified as ordinal values. Greater rate of high perceived stress was observed in the No-COVID sample (No-COVID: $17.65 \%$ vs. COVID: 6.90\%). Despite these data, DASS-21 stress score (COVID: $17.24 \%$ vs. No-COVID $5.88 \%$ ) was found to be as expected from the distribution of the values, as mentioned before. The same result was reported for DASS-21 anxiety (COVID: $13.79 \%$ vs. NoCOVID 11.76\%) and DASS-21 depression score showed similar results (COVID: $17.24 \%$ vs. No-COVID 17.65\%). All Distributions of DASS-21 stress, anxiety, depression and PSS scores are reported in Figure 1.

District prevalence. Due to the characteristic pattern of COVID outbreak in Italy, we divided the HCWs according to COVID-19 DP. Following the first 2 imported cases in Rome, the first Italian COVID-19 transmission was reported in Lombardy and higher prevalence was reported in the North of Italy (Lombardy, Veneto and Emilia Romagna) (3). Accordingly, Campus Bio-Medico University Hospital and Tor Vergata University Hospital were classified as low prevalence (low prevalence district) and Modena University Hospital together with Piacenza Parma Hospital as high prevalence (high prevalence district). Distribution of HCWs according to DP resulted in $19(43.30 \%)$ HCWs in the high prevalence district while $17(56.70 \%)$ questionnaires were collected from the control group (low prevalence group). Table VII displays the median scores and category distribution for DASS-21 stress, anxiety and PSS scores according to DP. According to our data, DASS-21 stress, anxiety and depression score distribution wasn't influenced by COVID-19 prevalence in the Hospital district $(p>0.05)$. Although the median value of the PSS score was higher in the high prevalence district, a statistically significant difference in terms of distribution was not found between the groups. However, a high rate of severe and extremely severe scores was found in DASS-21 subsets (DASS-21 stress $21.05 \%$ vs. $7.4 \%$; DASS-21 anxiety $26.32 \%$ vs. $3.7 \%$; DASS-21 depression $26.32 \%$ vs. $11.11 \%$ ). The same disposition was found for PSS scores of medium and high perceived stress $(73.86 \%$ vs. $62.96 \%)$. Figure 2 summarizes DSS-21 and PSS-10 distributions according to district prevalence (High vs. Low).

\section{Discussion}

Breast Cancer guidelines determined a shift in the clinical and health organization in the last 20 years, resulting in better clinical outcomes for patients (18-20). In order to provide the best clinical outcome, all surgeons and physicians involved in breast cancer care spent more than 
Table V. DAS-21 score, PSS score and work. All values are express as medians and IQRs (interquartile ranges). All categorical data are expressed as numbers, $p$-Values are obtained with One-way ANOVA for continuous variable and with Independent-Samples Kruskal-Wallis test for ordinal data.

\begin{tabular}{|c|c|c|c|c|}
\hline & Physician $(n=17)$ & Surgeon $(n=18)$ & Nurse $(n=10)$ & $p$-Value \\
\hline DASS-21 stress & $17.5[9.5 ; 20.5]$ & $17[11 ; 22]$ & $15[10 ; 34.5]$ & 0.307 \\
\hline DASS-21 anxiety & $14[12 ; 17.5]$ & $5[4 ; 9.5]$ & $5[2.5 ; 18]$ & 0.602 \\
\hline DASS-21 depression & $5[2 ; 11]$ & $6[4 ; 10]$ & $6[4 ; 32.5]$ & 0.247 \\
\hline PSS & $16[12.25 ; 19.5]$ & $17.5[12 ; 14]$ & $18[14 ; 25]$ & 0.348 \\
\hline \multicolumn{5}{|l|}{ DASS-21 stress } \\
\hline Normal & $5(29.45 \%)$ & $4(22.22 \%)$ & $4(40 \%)$ & \multirow[t]{5}{*}{0.711} \\
\hline Mild & $6(35.30 \%)$ & $10(55.56 \%)$ & $2(20 \%)$ & \\
\hline Moderate & $5(29.42 \%)$ & $2(11.12 \%)$ & $1(10 \%)$ & \\
\hline Severe & $1(5.83 \%)$ & $1(5.56 \%)$ & $0(0 \%)$ & \\
\hline Extremely severe & $0(0 \%)$ & $1(5.56 \%)$ & $3(3 \%)$ & \\
\hline \multicolumn{5}{|l|}{ DASS-21 anxiety } \\
\hline Normal & $10(58.82 \%)$ & $12(66.67 \%)$ & $6(60 \%)$ & \multirow[t]{5}{*}{0.966} \\
\hline Mild & $2(11.76 \%)$ & $1(5.56 \%)$ & $0(0 \%)$ & \\
\hline Moderate & $5(29.42 \%)$ & $2(11.12 \%)$ & $1(10 \%)$ & \\
\hline Severe & $0(0 \%)$ & $1(5.56 \%)$ & $0(0 \%)$ & \\
\hline Extremely severe & $0(0 \%)$ & $2(11.12 \%)$ & $2(20 \%)$ & \\
\hline \multicolumn{5}{|l|}{ DASS-21 depression } \\
\hline Normal & $11(64.70 \%)$ & $10(55.56 \%)$ & $7(70 \%)$ & \multirow[t]{5}{*}{0.735} \\
\hline Mild & $2(11.76 \%)$ & $3(16.67 \%)$ & $0(0 \%)$ & \\
\hline Moderate & $1(5.88 \%)$ & $2(11.12 \%)$ & $2(20 \%)$ & \\
\hline Severe & $2(11.76 \%)$ & $2(11.12 \%)$ & $0(0 \%)$ & \\
\hline Extremely severe & $1(5.88 \%)$ & $1(5.56 \%)$ & $1(10 \%)$ & \\
\hline \multicolumn{5}{|l|}{ PSS } \\
\hline Low perceived stress & 6 & 6 & $2(20 \%)$ & \\
\hline Medium perceived stress & $10(58.82 \%)$ & $10(55.56 \%)$ & $6(60 \%)$ & \\
\hline High perceived stress & $1(5.88 \%)$ & $2(11.12 \%)$ & $2(20 \%)$ & \\
\hline
\end{tabular}

$50 \%$ of their working hours with minimum caseload $(20,21)$. Breast Cancer treatment requires a profound knowledge of cancer behavior in order to reduce the risk of tumors progression (22-24). On the other hand, a surgical plan should be seen as an opportunity for breast remodeling that may enhance the woman's appearance (25-30). For these reasons, BCC HCWs' workload may be assumed as homogenous in terms of effect by perceived stress, anxiety and depression.

Resource reallocation and risk of cross infection are two of the many issues that BCC HCWs are facing during the COVID-19 outbreak. Today, having no reliable data regarding the end of the epidemic, the idea of postponing all treatments until the end of the outbreak is not feasible for cancer patients. Therefore, several guidelines have been published in order reduce the detrimental effect of COVID19 outbreak $(2,7,8,31-33)$. Triage of urgent clinical cases, awake and fast track surgery could increase the number of treated patients, reducing hospitalizations as well as the risk of cross-infection during the COVID-19 era (8, 34-38). Breast oncological treatment was only partially impaired by the COVID-19 outbreak and breast specialists try to provide the best cancer treatment for a higher number of patients $(2$, $7,8,35,39)$. Hence, breast cancer specialists could provide a good model for understanding how the COVID-19 outbreak could influence health well-being among the low risk HCWs population.

In our study, age did not show any correlation with DASS21 stress, anxiety, depression and PSS scores (R: 0.0217, $0.033,0.2424,0.1853$, respectively), as already demonstrated by Styra et al. in SARS outbreak (10).

In contrast to earlier findings determining that nurses are at higher risk of emotional distress in tertiary care hospital during outbreak $(10,12)$, our data demonstrated no difference in terms of DASS-21 and PSS scores between the different HCWs (physician, surgeon and nurse) in low risk environment. Although our results seem to contradict the data from Lai et al. and Styra et al., we underline that both works included HCWs directly exposed to index outbreak $(10,12)$. As stated before, our population consisted of Breast Cancer Center workers yet no one in the cohort assisted in any circumstances COVID-19 symptomatic patients. Thus, were considered at lower risk for COVID exposure. 
Table VI. DAS-21 score and PSS score according to COVID Hospital/NoCOVID Hospital. All values are expressed as medians and IQRS (interquartile ranges). All categorical data are expressed as numbers and percentages. $p$-Values were obtained with $t$-test for continuous variable and with Independent-Samples Mann-Whitney U-test for ordinal data.

\begin{tabular}{|c|c|c|c|}
\hline & $\begin{array}{c}\text { COVID } \\
\text { hospital } \\
(n=29)\end{array}$ & $\begin{array}{c}\text { No-COVID } \\
\text { hospital } \\
(\mathrm{n}=17)\end{array}$ & $p$-Value \\
\hline DASS-21 stress & $18[12 ; 24]$ & $14[10 ; 16]$ & 0.043 \\
\hline DASS-21 anxiety & $6[4 ; 10]$ & $4[2 ; 12]$ & 0.531 \\
\hline DASS-21 depression & $6[4 ; 20]$ & $8[4 ; 12]$ & 0.358 \\
\hline PSS-10 & $16[11 ; 19]$ & $17[13 ; 22]$ & 0.278 \\
\hline \multicolumn{4}{|l|}{ DASS-21 stress } \\
\hline Normal & $7(24.14 \%)$ & $6(35.29 \%)$ & 0.087 \\
\hline Mild & $9(31.03 \%)$ & $9(52.94 \%)$ & \\
\hline Moderate & $8(27.59 \%)$ & $1(5.88 \%)$ & \\
\hline Severe & $2(6.90 \%)$ & $0(0 \%)$ & \\
\hline Extremely severe & $3(10.34 \%)$ & $1(5.88 \%)$ & \\
\hline \multicolumn{4}{|l|}{ DASS-21 anxiety } \\
\hline Normal & $17(58.62 \%)$ & $11(64,70 \%)$ & 0.697 \\
\hline Mild & $3(10.34 \%)$ & $1(5.88 \%)$ & \\
\hline Moderate & $5(17.24 \%)$ & $3(17.65 \%)$ & \\
\hline Severe & $0(0 \%)$ & $1(5.88 \%)$ & \\
\hline Extremely severe & $4(13.79 \%)$ & $1(5.88 \%)$ & \\
\hline \multicolumn{4}{|l|}{ DASS-21 depression } \\
\hline Normal & $19(65.51 \%)$ & $10(58.82 \%)$ & 0.654 \\
\hline Mild & $3(10.34 \%)$ & $2(11.76 \%)$ & \\
\hline Moderate & $2(6.90 \%)$ & $2(11.76 \%)$ & \\
\hline Severe & $3(10.34 \%)$ & $1(5.88 \%)$ & \\
\hline Extremely severe & $2(6.90 \%)$ & $2(11.76 \%)$ & \\
\hline \multicolumn{4}{|l|}{ PSS } \\
\hline Low perceived stress & $10(34.48 \%)$ & $5(29.41 \%)$ & 0.456 \\
\hline Medium perceived stress & $17(58.62 \%)$ & $9(52,94 \%)$ & \\
\hline High perceived stress & $2(6.90 \%)$ & $3(17.65 \%)$ & \\
\hline
\end{tabular}

Bold value indicates statistical significance.

Neither sex among our population seemed to have an impact on the distribution of DAS-21 and PSS scores' higher values. In our cohort, women experienced median lower value of DASS-21 and higher value of PSS-10, without reaching statistical significance. These data are also in contrast with previous evidence which included partially or totally exposed COVID-19 or SARS HCWs $(10,12)$, but are in line with data from Goulia et al. during the A/H1N1 pandemic in a general hospital (9).

During the COVID-19 outbreak, the NHS reallocation of resources toward COVID-19 patients resulted in reduced availability of facilities, and even operatory theatres were transformed into Intensive Care Units $(1,2,8)$. Moreover, the Health system planned the creation of COVID hospitals, totally or partially dedicated to treatment of COVID-19 patients (40). Due to the scarcity of facilities and the fear of cross-infection, we hypothesized that low risk HCWs could suffer in terms of psychosocial well-being when compared
Table VII. DAS-21 score and PSS score according to District Prevalence. All values are express as medians and IQRs (interquartile ranges). All categorical data are expressed as numbers and percentages. $p$-Values were obtained with $t$-test for continuous variable and with independent-samples Mann-Whitney U-test for ordinal data.

\begin{tabular}{|c|c|c|c|}
\hline & $\begin{array}{c}\text { High } \\
\text { prevalence } \\
\text { district } \\
(\mathrm{n}=19)\end{array}$ & $\begin{array}{c}\text { Low } \\
\text { prevalence } \\
\text { district } \\
(\mathrm{n}=27)\end{array}$ & $p$-Value \\
\hline DASS-21 stress & $14[10 ; 21]$ & $16[11 ; 21]$ & 0.723 \\
\hline DASS-21 anxiety & $6[4 ; 14]$ & $4[2 ; 9]$ & 0.113 \\
\hline DASS-21 depression & $6[6 ; 20]$ & $6[4 ; 10]$ & 0.123 \\
\hline PSS-10 & $17[13 ; 21.5]$ & $16[9 ; 20]$ & 0.139 \\
\hline \multicolumn{4}{|l|}{ DASS-21 stress } \\
\hline Normal & $6(31.58 \%)$ & $7(25.93 \%)$ & 1.000 \\
\hline Mild & $7(36.84 \%)$ & $11(40.74 \%)$ & \\
\hline Moderate & $2(10.53 \%)$ & $7(25.93 \%)$ & \\
\hline Severe & $1(5.26 \%)$ & $1(3,70 \%)$ & \\
\hline Extremely severe & $3(15.79 \%)$ & $1(7.70 \%)$ & \\
\hline \multicolumn{4}{|l|}{ DASS-21 anxiety } \\
\hline Normal & $11(57.89 \%)$ & $17(3.70 \%)$ & 0.394 \\
\hline Mild & $1(5.26 \%)$ & $3(11.11 \%)$ & \\
\hline Moderate & $2(10.53 \%)$ & $6(22.22 \%)$ & \\
\hline Severe & $1(5.26 \%)$ & $0(0 \%)$ & \\
\hline Extremely severe & $4(21.05 \%)$ & $1(3.70 \%)$ & \\
\hline \multicolumn{4}{|l|}{ DASS-21 depression } \\
\hline Normal & $11(57.89 \%)$ & $18(3.70 \%)$ & 0.387 \\
\hline Mild & $2(10.53 \%)$ & $3(11.11 \%)$ & \\
\hline Moderate & $1(5.26 \%)$ & $3(11.11 \%)$ & \\
\hline Severe & $2(10.53 \%)$ & $2(7.41 \%)$ & \\
\hline Extremely severe & $3(15.80 \%)$ & $1(3.70 \%)$ & \\
\hline \multicolumn{4}{|l|}{ PSS } \\
\hline Low perceived stress & $5(26.31 \%)$ & $10(37.04 \%)$ & 0.170 \\
\hline Medium perceived stress & $10(52.63 \%)$ & $16(59.23 \%)$ & \\
\hline High perceived stress & $4(5.26 \%)$ & $1(3.70 \%)$ & \\
\hline
\end{tabular}

to no-COVID HCWs. Despite higher value of DASS-21 anxiety, no statistically significant distribution was found between the two populations and higher values of DASS-21 depression and PSS score were found in No-COVID HCWs. Interestingly, DASS-21 stress score values demonstrated a statistically significant distribution $(p=0.043)$ between COVID and No-COVID hospital, yet no difference was found when populations were grouped according to ordinal variables (normal, mild, moderate, severe, extremely severe).

Similar results were obtained when the population was divided according to DP. Following two imported cases in Rome, the COVID-19 Italian outbreak emerged mainly in the northern region, determining a relevant difference in terms of cases and fatality rate across the country (3). In order to assess the impact of prevalence differences on psychosocial well-being, we divided our cohort according to the number of cases in the hospital district. High prevalence district HCWs experienced higher median value of DAS-21 

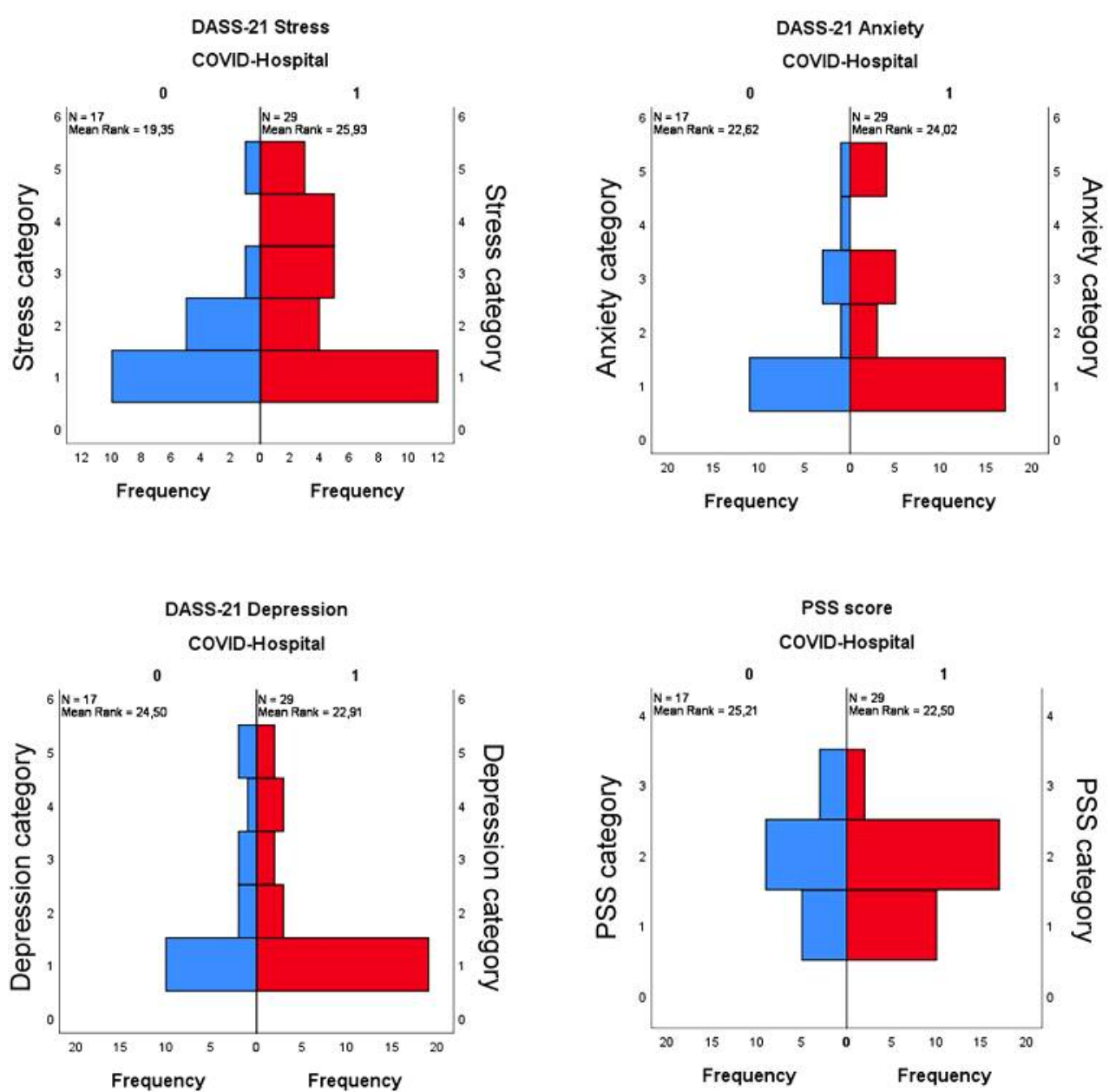

Figure 1. DASS-21 stress, anxiety, depression and PSS score Categories. COVID-Hospital 0: No; 1: Yes. For DASS-21 score 1: Normal, 2: Mild, 3: Moderate, 4: Severe, 5: Extremely Severe. PSS Score 1: Low Perceived Stress, 2: Medium Perceived Stress, 3: High Perceived Stress.

stress, anxiety, depression and PSS scores. Despite these results, no statistically significant difference was found between the groups (high vs. low prevalence). Furthermore, the same result was confirmed when both cohorts were divided according to score level: despite greater rate of higher level of stress, anxiety and depression in the high prevalence population, our study failed to demonstrate a statistically significant difference between HCWs among the different districts of prevalence in terms of psychosocial well-being.

In line with these data, careful measures of addressing psychosocial well-being during an outbreak should be taken by the hospital despite its role during a future outbreak or the incidence of future epidemic in the hospital district. In our opinion, these data obtained from BCC HCWs should be considered, especially for HCWs who are at low risk of infection.
Our study has the limitation of the small cohort, yet population baseline data were well matched when the population was grouped according to COVID-hospital/notCOVID-hospital and COVID-19 prevalence. Moreover, the Study was designed to have 4 different certified Breast Units. The choice of BCC was made in order to obtain data from different facilities in terms of COVID-19 treatment (COVID-hospital) and COVID-19 prevalence. However, further vaster studies with a larger cohort are required for the validation of these preliminary data. Furthermore, no data were available for the period before the COVID-19 outbreak and no comparison was made with this baseline period. This limitation was mainly due to the unexpected event of the COVID-19 outbreak. We decided not to collect retrospective data of pre-COVID-19 era. If confirmed in larger series, our data show that district prevalence differences do not affect psychological well-being of low risk HCWs during the 

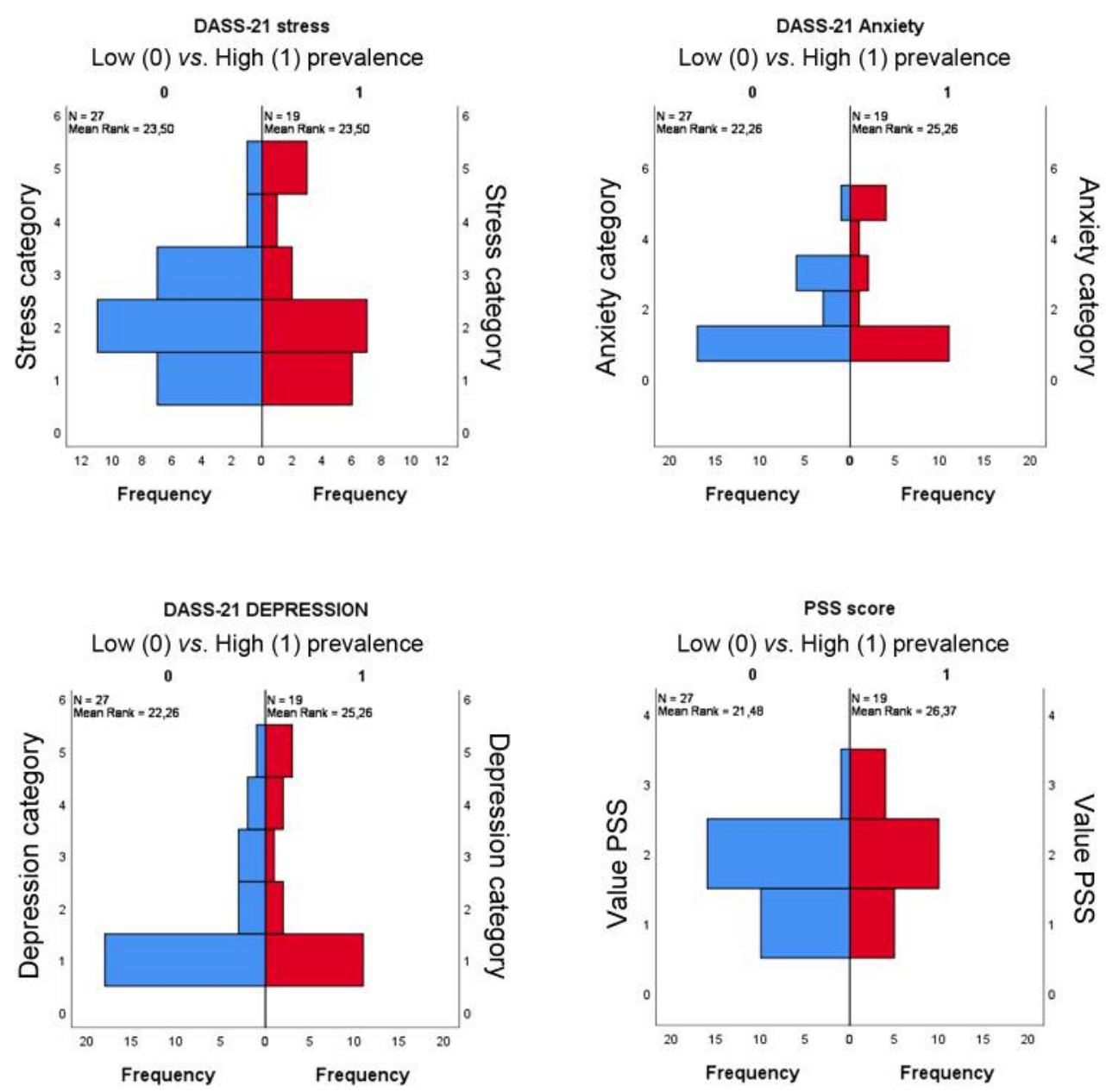

Figure 2. DASS-21 stress, anxiety, depression and PSS score categories divided according to prevalence district. Low prevalence district:0; high prevalence district:1. For DASS-21 score 1: Normal, 2: Mild, 3: Moderate, 4: Severe, 5: Extremely Severe. PSS Score 1: Low perceived stress, 2: Medium perceived stress, 3: High perceived stress.

COVID-19 pandemic. Conversely, COVID hospital HCWs seem to obtain a higher value of DASS-21 score when compared with No-COVID HCWs, regardless of the COVID-19 district prevalence. Further studies are needed in order to address the long-term effect of COVID-19 pandemic on HCWs, particularly among low risk HCWs. These topics are deferred to future work.

Our multicentric descriptive study demonstrated that working in a COVID-19 tertiary care institution did not affect the risk of perceived anxiety and depression symptoms among low risk HCWs during the COVID-19 pandemic and according to the COVID-19 prevalence.

Despite the absence of differences in the abovementioned data, COVID hospital HCWs experienced a statistically significant higher value of DASS-21 anxiety score, underlining the influence of hospital organization on HCWs' anxiety. In our Opinion, NHS and hospital administration should promote and provide psychological support to HCWs despite the COVID-19 prevalence in order to reduce the epidemic impact on all HCWs. Moreover, hospital administrations should take into special consideration the psychological support for HCWs in COVID Hospitals, regardless of their role in COVID management.

\section{Conflicts of Interest}

The Authors declare no conflicts of interest regarding this study.

\section{Authors' Contributions}

Study conception and design: Vanni Gianluca, Santori Francesca, Pellicciaro Marco, Buonomo Oreste Claudio; Collection of data-Tor Vergata Hospital: Cotesta Maria, De Majo Adriano; Collection of data-Campus Biomedico Hospital: Orsaria Paolo; Collection of data- Piacenza Parma Hospital: Cattadori Francesca; Collection of 
data-Modena University Hospital: Tazzioli Giovanni; Collection of data-project coordinator: Meucci Rosaria, Lamacchia Feliciana, Assogna Massimo; Analysis of data: Vanni Gianluca, Altomare Vittorio, Buonomo Oreste Claudio, Tazzioli Giovanni; Interpretation of data: Vanni Gianluca, Materazzo Marco, Chiaravalloti Agostistino; Article draft: Marco Materazzo, Gianluca Vanni, Caspi Jonathan; Critical revision: D'Angelillo Rolando Maria, Barbarino Rosaria, Pistolese Chiara Adriana, Perretta Tommaso, Chiocchi Marcello, Granai Alessandra Vittoria; Critical revision of literature: Ingallinella Sara, Morando Ljuba, Dalli Stefania, Portarena Ilaria.

\section{Acknowledgements}

This study was funded with the non-conditional contribution of the Italian Ministry of Health.

\section{References}

1 Rosenbaum L: Facing COVID-19 in Italy - ethics, logistics, and therapeutics on the epidemic's front line. N Engl J Med 382(20): 1873-1875, 2020. PMID: 32187459. DOI: 10.1056/NEJMp2005492

2 Vanni G, Pellicciaro M, Materazzo M, Palombi L and Buonomo OC: Breast cancer diagnosis in COVID19-era: Alert from Italy. Front Oncol 10: 938, 2020. DOI: 10.3389/fonc.2020.00938

3 Ministry of Health: Covid-19 - Situazione in Italia. Available from: http://www.salute.gov.it/portale/nuovocoronavirus/dettaglioContenu tiNuovoCoronavirus.jsp?lingua $=$ italiano $\& i d=5351 \&$ area $=$ nuovoCo ronavirus\&menu=vuoto [Last accessed March 21, 2020].

4 Porcheddu R, Serra C, Kelvin D, Kelvin N and Rubino S: Similarity in case fatality rates (CFR) of COVID-19/SARSCOV-2 in Italy and China. J Infect Dev Ctries 14, 2020. PMID: 32146445. DOI: $10.3855 /$ jidc. 12600

5 Adhikari SP, Meng S, Wu YJ, Mao YP, Ye RX, Wang QZ, Sun C, Sylvia S, Rozelle S, Raat H and Zhou H: Epidemiology, causes, clinical manifestation and diagnosis, prevention and control of coronavirus disease (COVID-19) during the early outbreak period: A scoping review. Infect Dis Poverty 9, 2020. PMID: 32183901. DOI: 10.1186/s40249-020-00646-x

6 Lau H, Khosrawipour V, Kocbach P, Mikolajczyk A, Schubert J, Bania $\mathrm{J}$ and Khosrawipour T: The positive impact of lockdown in Wuhan on containing the COVID-19 outbreak in China. J Travel Med, 2020. PMID: 32181488. DOI: $10.1093 / \mathrm{jtm} / \mathrm{taaa037}$

7 Vanni G, Materazzo M, Pellicciaro M, Ingallinella S, Rho M, Santori F, Cotesta M, Caspi J, Makarova A, Pistolese CA and Buonomo OC: Breast cancer and COVID-19: The effect of fear on patients' decision-making process. In Vivo 34(3), 2020.

8 Buonomo OC, Materazzo M, Pellicciaro M, Caspi J, Piccione E and Vanni G: Tor Vergata University-Hospital in the beginning of COVID-19-Era: Experience and recommendation for breast cancer patients. In Vivo 34(3), 2020.

9 Goulia P, Mantas C, Dimitroula D, Mantis D and Hyphantis T: General hospital staff worries, perceived sufficiency of information and associated psychological distress during the A/H1N1 influenza pandemic. BMC Infect Dis 10: 322, 2010. PMID: 21062471. DOI: 10.1186/1471-2334-10-322

10 Styra R, Hawryluck L, Robinson S, Kasapinovic S, Fones C and Gold WL: Impact on health care workers employed in high-risk areas during the Toronto SARS outbreak. J Psychosom Res 64: 177-183, 2008. PMID: 18222131. DOI: 10.1016/j.jpsychores. 2007.07 .015
11 Maunder RG, Lancee WJ, Rourke S, Hunter JJ, Goldbloom D, Balderson K, Petryshen P, Steinberg R, Wasylenki D, Koh D and Fones CSL: Factors associated with the psychological impact of severe acute respiratory syndrome on nurses and other hospital workers in Toronto. Psychosom Med 66: 938-942, 2004. PMID: 15564361. DOI: 10.1097/01.psy.0000145673.84698.18

12 Nickell LA, Crighton EJ, Tracy CS, Al-Enazy H, Bolaji Y, Hanjrah S, Hussain A, Makhlouf $S$ and Upshur REG: Psychosocial effects of SARS on hospital staff: Survey of a large tertiary care institution. CMAJ 170: 793-798, 2004. PMID: 14993174. DOI: $10.1503 / \mathrm{cmaj} .1031077$

13 Cohen S, Tyrrell DA and Smith AP: Negative life events, perceived stress, negative affect, and susceptibility to the common cold. J Pers Soc Psychol 64: 131-140, 1993. PMID: 8421249. DOI: 10.1037//0022-3514.64.1.131

14 Jiang LC, Yan YJ, Jin ZS, Hu ML, Wang L, Song Y, Li NN, Su $\mathrm{J}, \mathrm{Wu} \mathrm{DX}$ and Xiao T: The depression anxiety stress Scale-21 in Chinese hospital workers: reliability, latent structure, and measurement invariance across genders. Front Psychol 11, 2020. PMID: 32351436. DOI: 10.3389/fpsyg.2020.00741

15 Lai J, Ma S, Wang Y, Cai Z, Hu J, Wei N, Wu J, Du H, Chen T, Li R, Tan H, Kang L, Yao L, Huang M, Wang H, Wang G, Liu $\mathrm{Z}$ and $\mathrm{Hu} \mathrm{S}$ : Factors associated with mental health outcomes among health care workers exposed to coronavirus disease 2019. JAMA Netw Open 3: e203976, 2020. PMID: 32202646. DOI: 10.1001/jamanetworkopen.2020.3976

16 Cohen S, Kamarck T and Mermelstein R: A global measure of perceived stress. J Health Soc Behav 24: 385-396, 1983. PMID: 6668417.

17 Cohen S, Janicki-Deverts D and Miller GE: Psychological stress and disease. J Am Med Assoc 298: 1685-1687, 2007. PMID: 17925521. DOI: 10.1001/jama.298.14.1685

18 Biganzoli L, Marotti L, Cardoso MJ, Cataliotti L, Curigliano G, Cuzick J, Goldhirsch A, Leidenius M, Mansel R, Markopoulos C, Wyld L and Rubio IT: European guidelines on the organisation of breast centres and voluntary certification processes. Breast Care 14: 359-365, 2019. PMID: 31933581. DOI: 10.1159/000503603

19 Wilson ARM, Marotti L, Bianchi S, Biganzoli L, Claassen S, Decker T, Frigerio A, Goldhirsch A, Gustafsson EG, Mansel RE, Orecchia R, Ponti A, Poortmans P, Regitnig P, Rosselli Del Turco M, Rutgers EJT, Van Asperen C, Wells CA, Wengström Y and Cataliotti L: The requirements of a specialist Breast Centre. Eur J Cancer 49: 3579-3587, 2013. PMID: 23968730. DOI: 10.1016/j.ejca.2013.07.017

20 Kesson EM, Allardice GM, George WD, Burns HJG and Morrison DS: Effects of multidisciplinary team working on breast cancer survival: Retrospective, comparative, interventional cohort study of 13722 women. BMJ 344, 2012. PMID: 22539013. DOI: 10.1136/bmj.e2718

21 Skinner KA, Helsper JT, Deapen D, Ye W and Sposto R: Breast cancer: Do specialists make a difference? Ann Surg Oncol 10: 606-615, 2003. PMID: 12839844. DOI: 10.1245/aso.2003.06.017

22 Buonomo OC, Caredda E, Portarena I, Vanni G, Orlandi A, Bagni C, Petrella G, Palombi L and Orsaria P: New insights into the metastatic behavior after breast cancer surgery, according to well-established clinicopathological variables and molecular subtypes. PLoS One 12: e0184680, 2017. PMID: 28922402. DOI: 10.1371 /journal.pone .0184680

23 Buonomo OC, Grasso A, Pistolese CA, Anemona L, Portarena I, Meucci R, Morando L, Deiana C, Materazzo M and Vanni G: 
Evaluation of concordance between histopathological, radiological and biomolecular variables in breast cancer neoadjuvant treatment. Anticancer Res 40: 281-286, 2020. PMID: 31892577. DOI: 10.21873/anticanres.13950

24 Orsaria P, Caredda E, Genova F, Materazzo M, Capuano I, Vanni G, Granai AV, de Majo A, Portarena I, Sileri P, Petrella G, Palombi L and Buonomo OC: Additional nodal disease prediction in breast cancer with sentinel lymph node metastasis based on clinicopathological features. Anticancer Res 38: 21092117, 2018. PMID: 29599329. DOI: 10.21873/anticanres.12451

25 Galimberti V, Vicini E, Corso G, Morigi C, Fontana S, Sacchini $\mathrm{V}$ and Veronesi P: Nipple-sparing and skin-sparing mastectomy: Review of aims, oncological safety and contraindications. Breast 34: S82-S84, 2017. PMID: 28673535. DOI: 10.1016/j.breast.2017

26 Calì Cassi L, Vanni G, Petrella G, Orsaria P, Pistolese C, Lo Russo G, Innocenti $\mathrm{M}$ and Buonomo $\mathrm{O}$ : Comparative study of oncoplastic versus non-oncoplastic breast conserving surgery in a group of 211 breast cancer patients. Eur Rev Med Pharmacol Sci 20: 2950-2954, 2016. PMID: 27460720.

27 Buonomo OC, Morando L, Materazzo M, Vanni G, Pistilli G, Palla L, Di Pasquali C and Petrella G: Comparison of round smooth and shaped micro-textured implants in terms of quality of life and aesthetic outcomes in women undergoing breast reconstruction: a single-centre prospective study. Updates Surg, 2020. PMID: 32062785. DOI: 10.1007/s13304-020-00721-w

28 Pukancsik D, Kelemen P, Újhelyi M, Kovács E, Udvarhelyi N, Mészáros N, Kenessey I, Kovács T, Kásler M and Mátrai Z: Objective decision making between conventional and oncoplastic breast-conserving surgery or mastectomy: An aesthetic and functional prospective cohort study. Eur J Surg Oncol 43: 303-310, 2017. PMID: 28069398. DOI: 10.1016/j.ejso.2016.11.010

29 Buonomo OC, Varvaras D, Montuori M, Vanni G, Venditti D, Elia S, Santurro L, Granai A V., Petrella G and Rossi P: Onestage immediate implant-based breast reconstruction, using biological matrices after conservative mastectomies: Preliminary experience of the University Hospital of Tor Vergata, Rome. Chir 28: 221-226, 2015.

30 Orsaria P, Varvaras D, Vanni G, Pagnani G, Scaggiante J, Frusone F, Granai AV, Petrella G and Buonomo OC: Nodal status assessment in breast cancer: Strategies of clinical grounds and quality of life implications. Int J Breast Cancer 2014: 469803, 2014. PMID: 24672730. DOI: 10.1155/2014/469803

31 Society of Surgical Oncology: Resource for management options of breast cancer during COVID-19., 2020. Available at: https://www.surgonc.org/wp-content/uploads/2020/03/BreastResource-during-COVID-19-3.30.20.pdf [Last accessed April 6, 2020].
32 Liu B, Ma F, Wang J, Fan Y, Mo $\mathrm{H}$ and $\mathrm{Xu}$ B: Health management of breast cancer patients outside the hospital during the outbreak of 2019 novel coronavirus disease. Zhonghua Zhong Liu Za Zhi 42: E002, 2020. PMID: 32100979. DOI: 10.3760/cma.j.cn112152-20200221-00110

33 Fadare O, Dadmanesh F, Alvarado-Cabrero I, Snyder R, Stephen Mitchell J, Tot T, Wang SA, Ghofrani M, Eusebi V, Martel M and Tavassoli FA: Lobular intraepithelial neoplasia [lobular carcinoma in situ] with comedo-type necrosis: A clinicopathologic study of 18 cases. Am J Surg Pathol 30: 1445-1453, 2006. PMID: 17063087. DOI: $10.1097 / 01$.pas.0000213290.58283.82

34 Mineo TC, Sellitri F, Vanni G, Gallina FT and Ambrogi V: Immunological and inflammatory impact of non-intubated lung metastasectomy. Int J Mol Sci 18, 2017. PMID: 28686211. DOI: 10.3390/ijms 18071466

35 Vanni G, Materazzo M, Perretta T, Meucci R, Anemona L, Buonomo C, Dauri M, Granai AV, Rho M, Ingallinella S, Tacconi F, Ambrogi V, Chiaravalloti A, Schillaci O, Petrella G and Buonomo OC: Impact of awake breast cancer surgery on postoperative lymphocyte responses. In Vivo 33: 1879-1884, 2019. PMID: 31662515 . DOI: 10.21873 /invivo.11681

36 Vanni G, Tacconi F, Sellitri F, Ambrogi V, Mineo TC and Pompeo E: Impact of awake videothoracoscopic surgery on postoperative lymphocyte responses. Ann Thorac Surg 90: 973-978, 2010. PMID: 20732526. DOI: 10.1016/j.athoracsur.2010.04.070

37 Ackerman RS, Hirschi M, Alford B, Evans T, Kiluk J V and Patel SY: Enhanced REVENUE after surgery? A coststandardized enhanced recovery pathway for mastectomy decreases length of stay. World J Surg 43: 839-845, 2019. PMID: 30456482. DOI: $10.1007 / \mathrm{s} 00268-018-4850-0$

38 Calì Cassi L, Biffoli F, Francesconi D, Petrella G and Buonomo $\mathrm{O}$ : Anesthesia and analgesia in breast surgery: the benefits of peripheral nerve block. Eur Rev Med Pharmacol Sci 21: 13411345, 2017. PMID: 28387892.

39 Tacconi F, Vanni G and Pompeo E: Systemic host response in awake thoracic surgery. In: Awake Thoracic Surgery. Bentham Science Publishers Ltd., pp. 19-33, 2012.

40 Wong J, Goh QY, Tan Z, Lie SA, Tay YC, Ng SY and Soh CR: Preparing for a COVID-19 pandemic: a review of operating room outbreak response measures in a large tertiary hospital in Singapore. Can J Anesth, 2020. PMID: 32162212. DOI: $10.1007 / \mathrm{s} 12630-020-01620-9$ 